\title{
A materials-science perspective on tackling COVID-19
}

Zhongmin Tang $\mathbb{1 0}^{1,13}$, Na Kong ${ }^{1,13}$, Xingcai Zhang ${ }^{2,13}$, Yuan Liu ${ }^{1}$, Ping Hü ${ }^{3}$, Shan $\mathrm{Mou}^{4}$, Peter Liljeström ${ }^{5}$, Jianlin Shi ${ }^{3}$, Weihong Tan (10,6,7, Jong Seung Kim ${ }^{8}$, Yihai Cao ${ }^{5}$, Robert Langer(iD) ${ }^{9,10}$, Kam W. Leong ${ }^{11,12}$, Omid C. Farokhzad ${ }^{1 凶}$ and Wei Tao ${ }^{1 凶}$

Abstract | The ongoing SARS-CoV-2 pandemic highlights the importance of materials science in providing tools and technologies for antiviral research and treatment development. In this Review, we discuss previous efforts in materials science in developing imaging systems and microfluidic devices for the in-depth and real-time investigation of viral structures and transmission, as well as material platforms for the detection of viruses and the delivery of antiviral drugs and vaccines. We highlight the contribution of materials science to the manufacturing of personal protective equipment and to the design of simple, accurate and low-cost virus-detection devices. We then investigate future possibilities of materials science in antiviral research and treatment development, examining the role of materials in antiviral-drug design, including the importance of synthetic material platforms for organoids and organs-on-a-chip, in drug delivery and vaccination, and for the production of medical equipment. Materials-science-based technologies not only contribute to the ongoing SARS-CoV-2 research efforts but can also provide platforms and tools for the understanding, protection, detection and treatment of future viral diseases.

The novel coronavirus SARS-CoV-2 causes coronavirus disease 2019 (COVID-19) and has spread around the world, leading to a global pandemic ${ }^{1}$. The number of confirmed cases has reached 27,486,960 and the number of deaths has risen to 894,983 across 200 countries (as of September 9, 2020) ${ }^{2}$. The Center for Systems Science and Engineering at Johns Hopkins University ${ }^{3}$ has reported an even higher infection rate, with 27,699,112 confirmed cases and 900,203 deaths, indicating highly efficient transmission at an exponentially increasing rate ${ }^{1}$. The data from the World Health Organization (WHO) suggest that SARS-CoV-2 has a case fatality rate of about $3.3 \%$, which is higher than that of the 1918 influenza pandemic (2\%) and the 1957 influenza pandemic $(0.6 \%)^{4}$. The rate of the spread of SARS-CoV-2 is 40 -fold higher than that of SARS-CoV', which makes it more difficult to control. Therefore, addressing the SARS-CoV-2 pandemic requires scientific collaborations across borders and disciplines ${ }^{5}$.

Viruses are able to adapt to changing environmental conditions and rapidly evolve ${ }^{6}$. Therefore, accurate, rapid and long-term detection strategies, efficient protection and frequent updating of treatments are needed to control the spread of viruses, which requires multidisciplinary solutions. In addition, only a global network can achieve coordinated detection, protection, containment and therapy. Materials science has long played a key role in the rapid response to emerging viral diseases by developing instruments that reveal the structures of viral proteins, by designing point-of-care devices for accurate and rapid detection and diagnosis ${ }^{7,8}$, and by formulating therapeutic treatment and vaccination strategies 9.

In this Review, we discuss the role of materials science in antiviral research and highlight the importance of collaborations with virologists, epidemiologists and clinicians in addressing the global pandemic caused by SARS-CoV-2 (REF. ${ }^{1}$ ). We examine viral transmission, infection and disease progression (FIG. 1a), and discuss viral protection, detection, treatment and vaccination from a materials-science viewpoint (FIG. 1 b). Finally, we investigate the future role of materials science in studying infectious viruses and in the treatment of viral diseases. Indeed, the emergence of a new pandemic that is potentially more severe than the SARS-CoV-2 pandemic is not a matter of if but when ${ }^{1,10}$. Therefore, universal platform technologies offered by materials science will also play a crucial role in the understanding, protection, diagnosis, treatment and prevention of future viral outbreaks.

\section{Materials science against viruses}

Materials science is important in all areas of antiviral research, including investigation of viral structure and biology, protection, detection, treatment and vaccination. 
Virus sequence and structure. The generation of efficient approaches to the protection, detection and treatment of viral diseases requires a thorough understanding of viral sequence, structure and transmission. Materials science offers important tools and technologies for viral research. For example, individual viral particles can be tracked in cells using confocal microscopy ${ }^{11,12}$ (FIG. 2a,b), allowing the dissection of the mechanism of infection. Gene sequencing provides the basis of antiviral-drug design and genomic-biomarker detection. Nanotechnology, for example, nanopore sequencing, has contributed to the speed and accuracy of next-generation gene-sequencing platforms ${ }^{13}$ (FIG. 2c). Based on the gene sequence, viral proteins can be synthesized and their $3 \mathrm{D}$ structure can be reconstructed at an atomic level using cryo-electron microscopy, X-ray crystallography ${ }^{14}$ or NMR spectroscopy ${ }^{15}$. However, X-ray crystallography requires a large number of small crystals, and large proteins are difficult to investigate by NMR spectroscopy, which also requires high protein concentration and stability. These limitations can be addressed by singleparticle cryo-electron microscopy ${ }^{16}$, which has enabled the determination of the structure of many viruses ${ }^{17}$, including the 3.5 - $\AA$-resolution structure of the $\mathrm{CoV}$ spike (S) glycoprotein of SARS-CoV-2, as well as monoclonal antibody affinities ${ }^{18}$. Importantly, towards the understanding of viruses, materials science has played a key role in developing and optimizing instrumental components to ensure the accuracy and sensitivity of characterizations.

Protection from viral infection. Viruses replicate inside living cells and are transmitted by direct contact ${ }^{19}$, saliva $^{20}$, semen, vaginal fluid, anal mucus ${ }^{21}$, air ${ }^{22}$, bloodsucking insects ${ }^{23}$, food ${ }^{24}$, blood exposure ${ }^{25}$ or from pregnant women to newborns. SARS-CoV-2 first attaches

\footnotetext{
Author addresses

${ }^{1}$ Center for Nanomedicine and Department of Anesthesiology, Brigham and Women's Hospital, Harvard Medical School, Boston, MA, USA.

${ }^{2}$ School of Engineering and Applied Sciences, Harvard University, Cambridge, MA, USA.

${ }^{3}$ State Key Laboratory of High Performance Ceramics and Superfine Microstructure, Shanghai Institute of Ceramics, Chinese Academy of Sciences, Shanghai, China. ${ }^{4}$ Institute of Molecular Medicine (IMM), Renji Hospital, State Key Laboratory of Oncogenes and Related Genes, School of Medicine, Shanghai Jiao Tong University, Shanghai, China.

${ }^{5}$ Department of Microbiology, Tumor and Cell Biology, Karolinska Institutet, Stockholm, Sweden.

${ }^{6}$ Molecular Science and Biomedicine Laboratory (MBL), State Key Laboratory of Chemo/Biosensing and Chemometrics, College of Chemistry and Chemical Engineering, College of Biology, Aptamer Engineering Center of Hunan Province, Hunan University, Changsha, China.

${ }^{7}$ The Cancer Hospital of the University of Chinese Academy of Sciences, Institute of Basic Medicine and Cancer (IBMC), Chinese Academy of Sciences, Hangzhou, China. ${ }^{8}$ Department of Chemistry, Korea University, Seoul, Korea.

${ }^{9}$ Koch Institute for Integrative Cancer Research, Massachusetts Institute of Technology, Cambridge, MA, USA.

${ }^{10}$ Department of Chemical Engineering, Massachusetts Institute of Technology,

Cambridge, MA, USA.

${ }^{11}$ Department of Biomedical Engineering, Columbia University, New York, NY, USA.

${ }^{12}$ Department of Systems Biology, Columbia University Irving Medical Center,

New York, NY, USA.

${ }^{13}$ These authors contributed equally: Zhongmin Tang, Na Kong, Xingcai Zhang.
}

to the host cell through binding of the S protein to the angiotensin-converting enzyme 2 (ACE2) receptor, allowing it to enter the cell and release its viral RNA (FIG. 1a). In the cell, the virus hijacks the machinery of the host cell to synthesize RNA and produce structural proteins, which assemble into new viruses that are released from the host cell. The replication cycle is then repeated ${ }^{26,27}$. Not only humans but also animals (cats, golden hamsters and ferrets) ${ }^{28,29}$ can be infected by SARS-CoV-2.

The most straightforward method for protection is physically isolating viruses by masks, gloves, face shields and protective suits. Surgical masks are made of three different fibre layers to prevent the entry of viruses; the outside layer is designed to stop liquid splash; the interlayer acts as a barrier against viruses and bacteria; and the inner coating absorbs moisture exhaled by the wearer ${ }^{30}$. Different materials can be synthesized to act as protective coatings, such as polyimide thin films with nanoporous membranes ${ }^{31}$ and carbon-based materials ${ }^{32}$. A polymer nanofibre filter, with the surface chemistry optimized to match that of particulate matter, allows both transparency to sunlight and sufficient airflow $^{33}$. A self-powered air filter composed of an ionic liquid-polymer composite and a sponge network also enables efficient removal of particulate matter, including nanoparticles $^{34}$ (FIG. 2d). Although these materials have not yet been used for viral protection owing to safety and cost concerns, they could be modified and introduced for virus protection in the foreseeable future, which will require research efforts to simplify synthesis procedures and guarantee safety.

Detection of infected patients. Rapid identification of infected patients helps limit the spread of viruses. Immunoassays and nucleic-acid-amplification tests using PCR are widely used and reliable methods for detecting viral proteins and nucleic acids, respectively. The accuracy of the results of immunoassays, such as radioimmunoassay ${ }^{35}$, enzyme-linked immunosorbent assay $^{36}$ and the immune colloidal gold technique ${ }^{37}$, depends on the sensitivity of the instruments and materials. The immune colloidal gold technique is an immunoassay based on labelling the antibody with plasmonic gold nanostructures, enabling a fluorescent readout once the antigen is captured by the antibody. This technique can be used for the detection of viral antigens, for example, of the Zika and dengue viruses ${ }^{7}$. Compared with conventional singleplex approaches, this multiplexed immunoassay can amplify near-infrared fluorescence by up to 100 times, substantially improving the imaging of antibody binding. In addition, the immune colloidal gold technique requires only $1 \mu$ of human serum, owing to its high sensitivity (FIC. 3a). Nanowire field-effect transistors can be combined with immune colloidal gold assays ${ }^{38}$ for real-time and direct electrical detection of single virus particles based on conductance changes after antigen-antibody binding (FIG. 3b). Similarly, an external electrical pulse can be applied to incorporate graphene quantum dots and gold-embedded polyaniline nanowires during the virus-accumulation step to increase the sensitivity of virus detection, for example, for the detection of the hepatitis E virus ${ }^{39}$ (FIG. 3C). 


\section{a Virus}

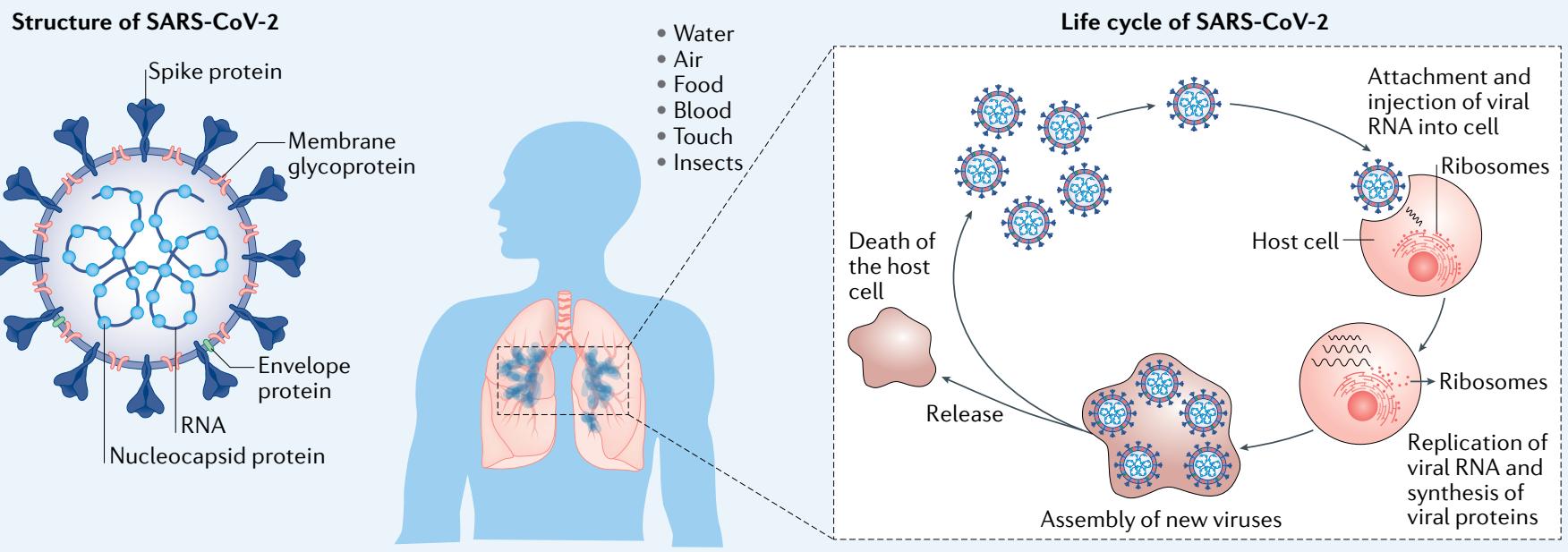

\section{b Materials science}

Understanding and protection
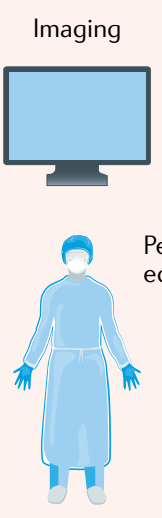

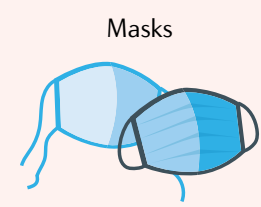

Personal protective equipment



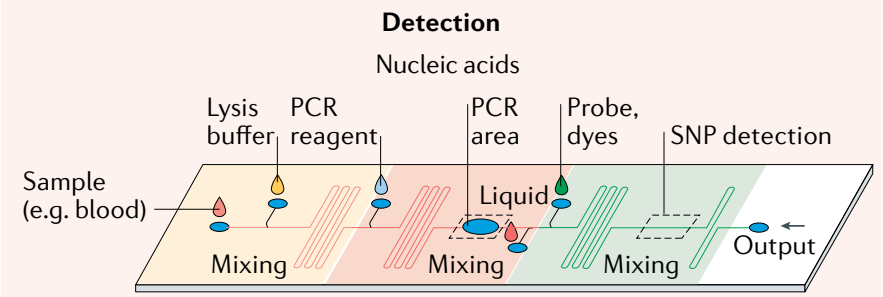

Extraction Amplification Detection

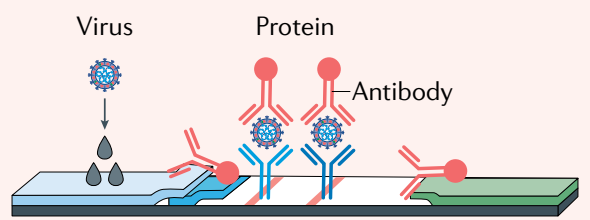

Treatment and vaccination
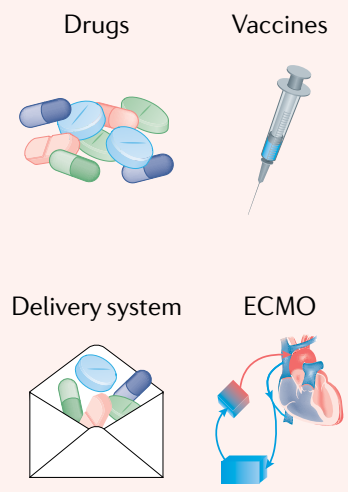

Fig. 1 | SARS-CoV-2 and materials science. a | The structure, transmission routes and replication cycles of SARS-CoV-2. b | Materials science contributes to the development and optimization of protective equipment and provides technologies and tools for the analysis of SARS-CoV-2, for example, high-resolution imaging, for sequencing (PCR) and protein analysis (immunoassays), for viral detection, for vaccine and treatment development and delivery, as well as by contributing advanced materials for clinical instruments, for example, filters for extracorporeal membrane oxygenation (ECMO) machines. SNP, single nucleotide polymorphism.

Real-time PCR with reverse transcription (rRT-PCR) is commonly used for the detection of viral DNA or $\mathrm{RNA}^{40}$, and is the recommended technique for the detection of the single-stranded RNA virus SARS-CoV-2 (REF $\left.{ }^{41}\right)$. However, PCR-based techniques for nucleic-acid detection come with trade-offs in terms of efficacy, cost, simplicity and speed. Alternatively, the CRISPR effectors Cas12 (REFS ${ }^{42,43}$ ) and Cas13a ${ }^{44}$ can be applied for nucleic-acid detection. For example, specific high-sensitivity enzymatic reporter unlocking (SHERLOCK) is a fluorescence-based or lateral-flow-based assay using CRISPR nucleases for nucleic-acid detection ${ }^{45}$. Interestingly, a prophylactic antiviral CRISPR strategy using Cas 13 has also been proposed to inhibit SARS-CoV-2 in human cells ${ }^{46}$. Single-molecule nucleic-acid sensitivity and selectivity can also be achieved using a biosensing platform based on microcavities. In this platform, the hybridization kinetics of matching and non-matching strands are detected by monitoring microcavity resonance wavelength shifts caused by interaction of the molecules with nanorods ${ }^{47}$ (FIG. 3d). This label-free technology has the advantage of transiently monitoring molecular interactions, allowing statistical analysis of interaction kinetics and increasing sensor lifetime. Nanopore-based platforms can also be used for targeted sequencing, and have already been applied for SARS-CoV-2 virus detection $^{48}$.

The immune system responds to viral infections and induces detectable symptoms, such as high body temperature. Non-contact detection of body temperature is mainly performed to preliminarily detect infected patients by using infrared thermal-imaging systems, for example, hand-held infrared temperature guns. However, the temperature gun is inaccurate at cold ambient temperatures, because the temperature is calculated based on a Seebeck coefficient ${ }^{49}$ close to the body temperature, which is not available at low temperatures. 




b Single-virus tracking

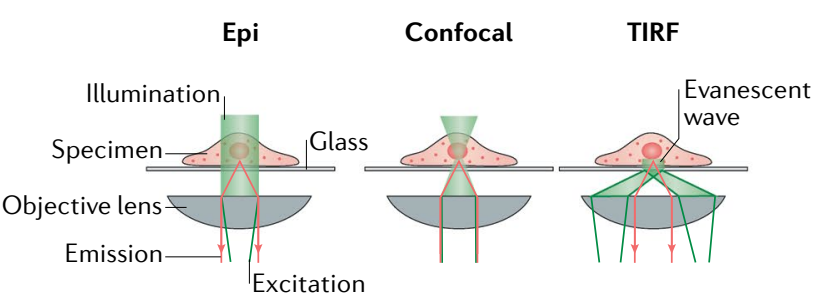

c Nanopore sequencing


Fig. 2 | Materials science in viral research and protection. a | Single-virus tracking workflow using fluorescence microscopy. The representative image shows an influenza virus in Chinese hamster ovary cells ( $z$-stacked time-lapse images, the colour code from pink/blue to yellow/white indicates the timescale from $0 \mathrm{~s}$ to $500 \mathrm{~s}) . \mathbf{b}$ | There are three possible imaging geometries in single-virus tracking, that is, epifluorescence geometry (Epi), confocal microscopy and total internal reflection fluorescence (TIRF) geometry. $\mathbf{c}$ | Nanopore sequencing using a nanosize pore and sensing regions in Mycobacterium smegmatis porin A (MspA) and a-haemolysin. $\mathbf{d}$ | A self-powered air filter can capture particulate matter and nanoparticles by surface adhesion. Arg, argon ion; BS, beam splitter; CCD, charge-coupled device; DM, dichroic mirror; F, filter; M, mirror; Nd:YAG, neodymium-doped yttrium aluminium garnet; S, shutters; SL, optical slits to control image size. Top image of panel a adapted with permission from REF. ${ }^{12}$, PNAS. Panels $\mathbf{a}$ and $\mathbf{b}$ reprinted from REF. ${ }^{11}$, Springer Nature Limited. Panel $\mathbf{c}$ reprinted from REF. ${ }^{13}$, Springer Nature Limited. Panel d reprinted from REF. ${ }^{34}$, CC BY 4.0.

Although laboratory assays have been developed for identifying infected patients, the detection and diagnosis of severe viral infections, such as COVID-19, may also require clinical diagnostic methods, such as computed tomography (CT), ultrasonic imaging, magnetic resonance imaging, single-photon emission computerized tomography and positron emission tomography. For example, CT can be used to detect interstitial changes and small patchy shadows in the lung, as well as extrapulmonary bands, in patients infected with SARS-CoV-2 (REF. $\left.{ }^{50}\right)$. However, instruments and professional medical staff are limited, and, therefore, portable diagnosis instruments ${ }^{51,52}$ and artificial intelligence $(\mathrm{AI})^{53}$ should be further developed and combined with clinical diagnosis to improve the detection process and guarantee the accuracy of results.

Treatment and vaccination. Currently available antiviral drugs can be broadly divided based on their mechanism of action into penetration and husking inhibitors, DNA polymerase inhibitors, reverse transcriptase inhibitors, protein inhibitors, neuraminidase inhibitors and broad-spectrum antiviral drugs ${ }^{54-56}$. Indeed, the majority of antiviral drugs currently used in the clinic are polymerase inhibitors, which are specific for a given viral enzyme. However, owing to their specificity, polymerase inhibitors cannot be applied for the treatment of infections with new viruses, such as SARS-CoV-2.

In contrast to bacteria, which can often be treated with antibiotics owing to their similar structure and replication mechanisms, the structure and replication of viruses greatly differ between types and species. Therefore, only few broad-spectrum antiviral drugs exist $^{57,58}$. For example, cyclodextrins modified with mercaptoundecane sulfonic acids mimic the key viricidal effects of heparan sulfates without causing systemic toxicity ${ }^{59}$. Alternatively, antiviral nanoparticles have been developed with flexible and long linkers that mimic heparan sulfate proteoglycans. The nanoparticles generate high forces $(\sim 190 \mathrm{pN})$, which irreversibly deform the virus $^{60}$ (FIG. 4a). Based on the spatially defined arrangement of ligands for the viral spike protein, bacteriophage capsids have also been designed that carry a multivalent binder. The bacteriophages can cover the entire virus 
envelope and, thus, prevent its binding to the host cell ${ }^{61}$. In addition, nanosponges derived from the plasma membranes of human lung epithelial type II cells or of human macrophages can neutralize SARS-CoV-2 by displaying the protein receptors necessary for SARS-CoV-2 entry into host cells ${ }^{62}$.

Similarly, biomimetic cell membrane vesicles can act as delivery platforms. Cell membrane vesicles have excellent biocompatibility and possess the inherited functions of the source cells, such as long circulation time (red blood cells) ${ }^{63}$, specific targeting moieties and receptors ${ }^{62,64}$. For example, virus-mimetic nanovesicles can deliver antiviral antigens and mimic the viral property to trigger specific immunogenicity ${ }^{65}$. Nanodecoys composed of cell membranes of the Zika virus and gelatin nanoparticles can be administered to capture the Zika virus and inhibit Zika virus entry and infection ${ }^{66}$. Nanomaterials may also be applied to control the cytokine storm associated with pneumonia, which is a common symptom in patients with severe a Virus detection by immune colloidal gold technique
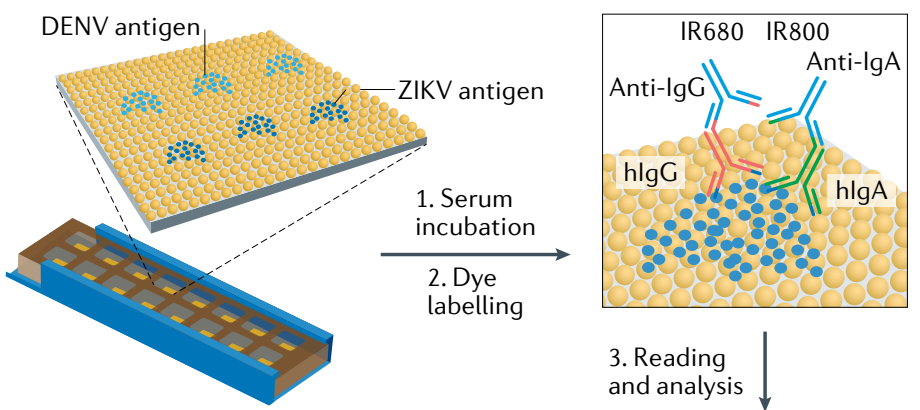

ZIKV infected DENV infected

Control


b Nanowire-based virus detection
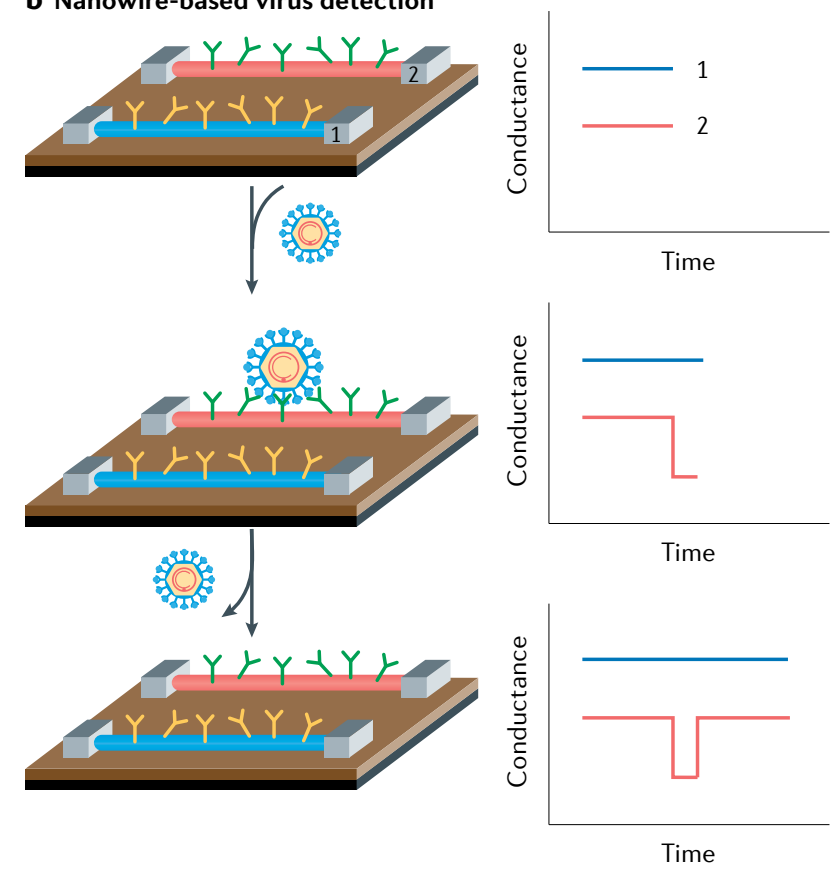

Time

d Plasmonic biosensor c GQD biosensor





Fig. 3 | Materials science in virus detection. a | Multiplexed Zika virus/ dengue virus (ZIKV/DENV) antigen microarray combining nanostructured plasmonic gold and near-infrared fluorescence molecules. Antibodies against ZIKV and DENV antigens in human serum are first captured by the microarray and then labelled with anti-human immunoglobulin G-infrared fluorescent dye 680 (IgG-IRDye680) and immunoglobulin A-infrared fluorescent dye 800 (IgA-IRDye800). Binding between $\lg \mathrm{G}$ and $\lg A$ with antigens is evaluated by measuring the fluorescence intensities of the two dyes. $\mathbf{b}$ | Nanowire-based detection of single viruses. Binding of the virus to a specific antibody (Ab) leads to a change in conductance. $\mathbf{c} \mid$ An external electrical pulse and biosensors based on graphene quantum dots (GQDs) and gold-embedded polyaniline nanowires (AuNP-PAni) can be used for the detection of hepatitis E virus (HEV). The biosensor electrode, which is based on anti-HEV Ab-conjugated to nitrogen and sulfur codoped graphene quantum dots and gold-embedded polyaniline nanowires (Ab-N,S-GQDs@AuNP-PAni), can capture HEV. The HEV concentration is determined from the pulse-induced impedimetric response. $\mathbf{d} \mid \mathrm{A}$ singlemolecule whispering gallery mode biosensor platform using plasmonic gold nanorods can be used to detect single nucleic acid molecules. EDC, 1-ethyl-3(3-dimethylaminopropyl) carbodiimide hydrochloride; GCE, glass carbon electrode; NHS, N-hydroxysuccinimide; N,S-GQDs, nitrogen and sulfur codoped graphene quantum dots; PBS, polarizing beam splitter; PD, photodetector; PDMS, polydimethylsiloxane. Panel a reprinted from REF. ${ }^{7}$, Springer Nature Limited. Panel $\mathbf{b}$ reprinted with permission from REF. ${ }^{38}$, PNAS. Panel $\mathbf{c}$ reprinted from REF. ${ }^{39}$, CC BY 4.0. Panel d reprinted from REF. ${ }^{47}$, Springer Nature Limited. 
a Antiviral AuNPs

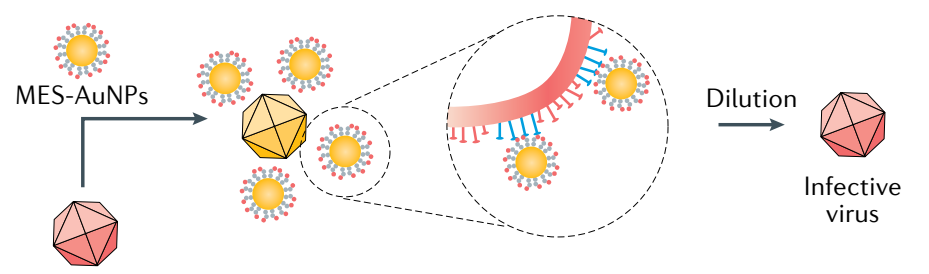

HSPG-binding

infective virus

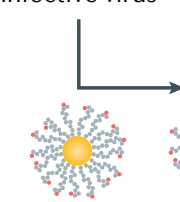

MUS-AuNPs

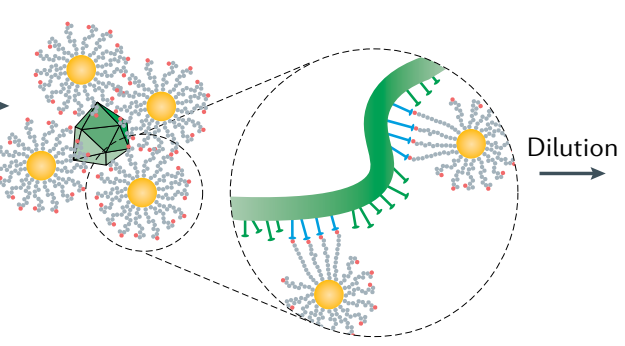

b Pulmonary surfactant biomimetic liposomes

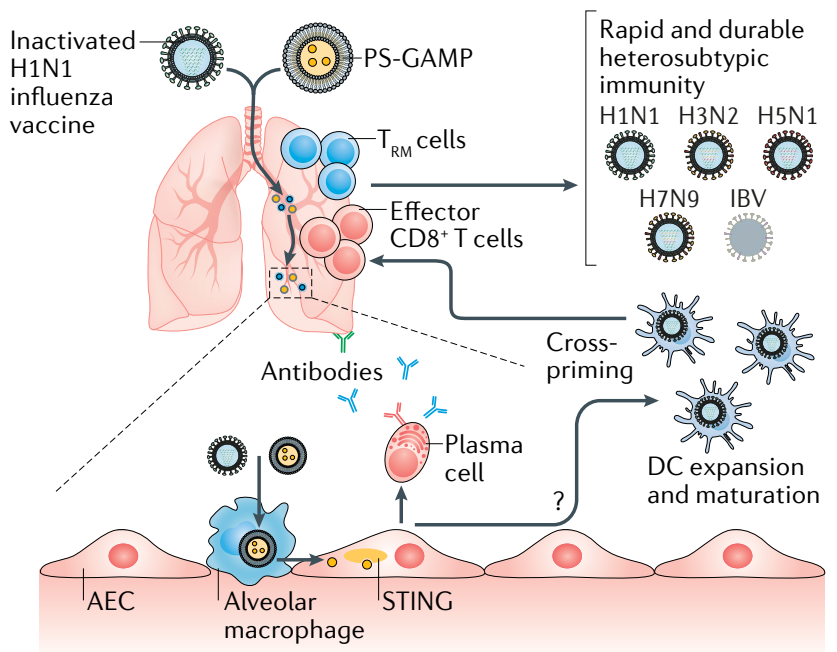

c Lipid nanoparticle-based vaccines

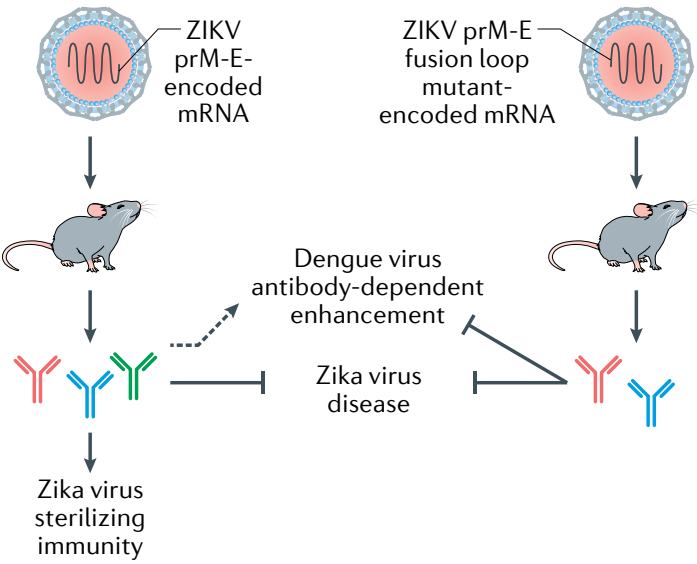

d Extracorporeal membrane oxygenation

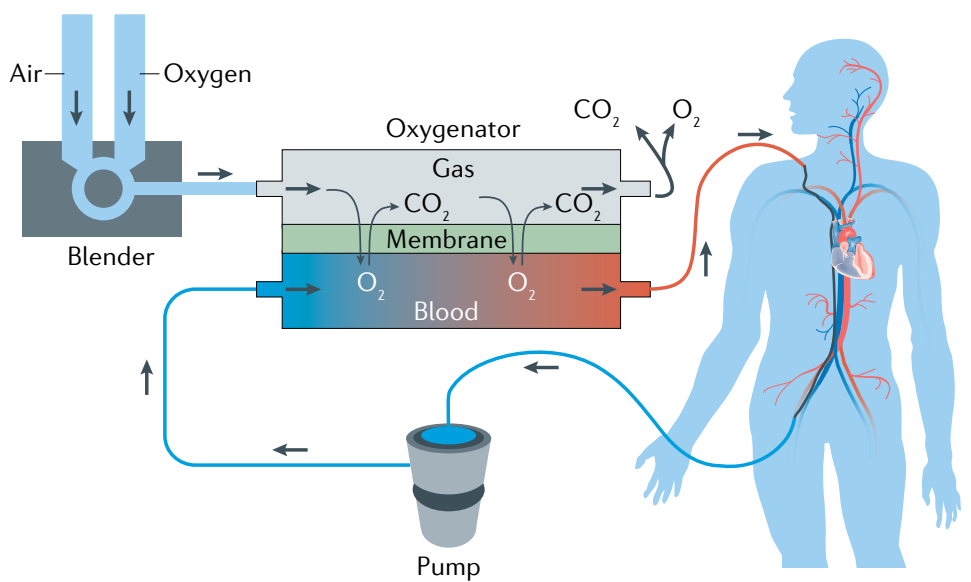

Fig. 4 | Materials science in the treatment and vaccination of viral diseases. a | Gold nanoparticles (AuNPs) coated with long and flexible moieties of undecanesulfonic acid (MUS) show viricidal activity against heparan sulfate proteoglycan (HSPG)-binding viruses, owing to the generation of high forces $(\sim 190 \mathrm{pN})$, which irreversibly deform the virus; by contrast, 3-mercaptoethylsulfonate (MES)-coated AuNPs are not antiviral because of the short molecule length. $\mathbf{b}$ | Nasal delivery of inactivated H1N1 influenza virus and pulmonary surfactant guanosine monophosphateadenosine monophosphate (PS-GAMP; an activator of stimulator of interferon genes (STING)) leads to the stimulation of dendritic cell (DC) maturation, antibody generation and, subsequently, to a $\mathrm{CD} 8^{+} \mathrm{T}$ cell and tissue-resident memory $T\left(T_{R M}\right)$ cell response, generating broad protection against seasonal influenza $B$ virus (IBV), $\mathrm{H} 3 \mathrm{~N} 2, \mathrm{H} 5 \mathrm{~N} 1$ and $\mathrm{H} 7 \mathrm{~N} 9$ influenza viruses. c | Lipid nanoparticles can be used for the delivery of a Zika virus pre-membrane and envelope (ZIKV prM-E)-encoding mRNA vaccine against the Zika virus. Delivering a ZIKV prM-E fusion loop mutant-encoding mRNA diminishes the generation of cross-reactive antibodies that promote Dengue virus infection. $\mathbf{d}$ | During extracorporeal membrane oxygenation, venous blood is drained from the body, oxygenated by fresh gas (the blender modulates the ratio between air and oxygen) using a gas-exchange device and then returned to the body. AEC, alveolar epithelial cell. Panel a reprinted from REF. ${ }^{60}$, Springer Nature Limited. Panel $\mathbf{b}$ from REF. ${ }^{84}$, Herold, S. \& Sander, L.-E. Toward a universal flu vaccine. Science $\mathbf{3 6 7}$, 852-853 (2020). Redrawn with permission from AAAS. Panel c reprinted with permission from REF. ${ }^{89}$, Elsevier. Panel d from REF. ${ }^{95}$, N. Engl. J. Med. Brodie, D. \& Bacchetta, M. Extracorporeal membrane oxygenation for ARDS in adults. 365, 1905-1914. Copyright ( $)$ (2011) Massachusetts Medical Society. Reprinted with permission from Massachusetts Medical Society.
COVID-19. For example, neutrophil membrane-coated poly(lactic-co-glycolic acid) nanoparticles can neutralize pro-inflammatory cytokines and suppress inflammation in inflammatory arthritis, which might also be applicable for pneumonia ${ }^{67}$.

The development of new antiviral drugs is challenging and requires thorough knowledge of the infection and replication mechanisms of the specific virus. For example, based on the genome sequence similarity between SARS-CoV and SARS-CoV-2, the serine protease TMPRSS2 was identified to be crucial for $S$ protein priming and, thus, for SARS-CoV-2 cell entry via ACE2. Therefore, a TMPRSS2 inhibitor may provide a treatment strategy to prevent SARS-CoV-2 infection ${ }^{68}$. Remdesivir, which is a nucleotide analogue prodrug that inhibits RNA-dependent RNA synthetase, has also been explored for COVID-19 treatment ${ }^{69}$. Preliminary data of the Randomised Evaluation of COVID-19 Therapy (RECOVERY) trial, testing dexamethasone as a treatment strategy for hospitalized COVID-19 patients, shows a lower 28-day mortality rate of treated patients than patients in the standard-care group, supporting a 
potential treatment effect of dexamethasone in patients who need respiratory support ${ }^{70}$. Injection or inhalation of dexamethasone nanoformulations may promote the treatment efficacy on COVID-19 through targeting of the drug to hyperactivated immune cells, potentiating its antioedema activity and antifibrotic effects ${ }^{71}$. Moreover, neutralizing antibodies ${ }^{72-74}$ and nanobodies (antibody fragments $)^{75,76}$ have been proposed for COVID-19 treatment. A recent clinical trial (NCT04313127), testing neutralizing antibodies to prevent COVID-19, had shown satisfactory results and is in further evaluation ${ }^{77,78}$. However, achieving a high concentration of neutralizing antibodies is crucial for efficacy and, thus, aluminium-based adjuvants, such as those used for PiCoVacc ${ }^{79,80}$, may be helpful.

Vaccines remain the most efficient strategy to protect against viral infections, because they generate specific antibodies ${ }^{81}$. Following infection, the virus causes inflammation and stimulates the release of chemokines, which attract leucocytes to the infected area to destroy the virus ${ }^{82}$. At the same time, phagocytes engulf viruses, disassemble them, deliver them to nearby lymph nodes and present the antigens to other immune cells, such as B and T cells, to initiate the production of antibodies that specifically recognize viral antigens ${ }^{82}$. However, upon first contact with a virus, the immune response is slow, and can only help patients to recover, but not to prevent the disease. Once antigen-specific $\mathrm{T}$ and $B$ cells are activated, they proliferate and generate memory cells. Therefore, upon second contact with the same type of virus, the immune system can respond quickly and efficiently. Pulmonary surfactant biomimetic liposomes can be applied to encapsulate and deliver an agonist of the interferon gene inducer, that is, stimulator of interferon genes, to increase the immune response against influenza viruses ${ }^{83,84}$ (FIG. 4b).

The purpose of a vaccine is to protect the body against a specific virus, capitalizing on the memory of the immune system, so that the immune system can eliminate the virus upon first contact. Vaccine design includes the identification of an antigen and adjuvant, which boosts the immune response caused by the antigen ${ }^{85}$, as well as an appropriate delivery platform. A variety of vaccines have been developed, including liveattenuated vaccines, inactivated vaccines, toxoid vaccines, subunit vaccines, conjugate vaccines, DNA vaccines, $m R N A$ vaccines and recombinant vector vaccines. $m R N A$ vaccines $^{86}$, which directly induce antigen expression, provide an interesting alternative for RNA viruses, such as the Zika virus and SARS-CoV-2, which are susceptible to mutation and, therefore, to drug resistance ${ }^{6}$. mRNA vaccines require nanocarriers for encapsulation and stabilization of the mRNA ${ }^{87,88}$; for example, mRNA can be encapsulated in lipid nanoparticles to protect mice against $\mathrm{Zika}^{89}$ virus infection (FIG. $4 \mathrm{C}$ ).

Although there is no mRNA vaccine yet approved for any disease, several clinical trials are under way. For example, Pfizer Inc., in a collaboration with BioNTech AG, is working on the phase II/III clinical trial of BNT162 mRNA vaccines for COVID-19 (NCT04368728) ${ }^{90}$ and has just announced data from preclinical studies ${ }^{91}$. Moderna, Inc. is currently conducting phase II clinical trials (NCT04232280) with an mRNA vaccine encoding a monoclonal antibody against cytomegalovirus ${ }^{92}$. In addition, the mRNA-1273 vaccine, developed by Moderna, Inc., against SARS-CoV-2 is currently in phase III clinical trials (NCT04470427) ${ }^{93}$. These mRNA vaccines are all delivered by lipid nanoparticles.

Finally, medical devices, such as ventilators, are important for the treatment of infected patients, to provide oxygen and ensure airflow in the lungs. Alternatively, extracorporeal membrane oxygenation (ECMO) can be applied in patients with severe respiratory failure to promote their recovery process ${ }^{94}$. In this procedure, venous blood is drained from the body, oxygenated using a gas-exchange device and then returned to the body ${ }^{95}$ (FIG. 4d). ECMO has also been applied for the treatment of COVID-19 patients $^{94}$.

\section{Possibilities for materials science}

Materials science has long played an important role in the study of viruses and in the treatment of viral infections ${ }^{18,37,44,96-106}$ (FIC. 5). In the future, materials science and engineering will certainly continue to contribute to confronting viral outbreaks by providing platform technologies and tools for virus research, ensuring efficient and comfortable protection, performing rapid, accurate and low-cost detection, and enabling timely and effective treatment and vaccination.

Physical protection. Physical protection provides a key strategy to prevent viral infection. Materials science can be applied to optimize physical-protection platforms in terms of preparation, manufacturing, material performance and structure, and to balance comfort and practicality. Commercial face masks can reduce virus emission from infected patients by providing a physical barrier with a certain cut-off size ${ }^{30}$. However, masks could also be designed to incorporate drugs or physical obstacles for killing the virus and stopping the transmission; for example, the virus could be damaged by heat generated by photothermal materials, such as graphene ${ }^{32}$, or by producing reactive oxygen species through a photodynamic process ${ }^{107-109}$. The design of reusable masks could further address personal protective equipment shortages, as experienced during the COVID-19 pandemic. Reusable masks would need to retain filtration ability and remain safe after decontamination, such as ultraviolet irradiation, moist heat and vaporous hydrogen peroxide ${ }^{110}$. To address this challenge, fibres, such as washable polypropylene fibres coated with assembled metal-organic frameworks ${ }^{111}$ or thermally stable electrospun polyimide/zeolitic imidazolate framework-8 nanofibres ${ }^{112}$, could be applied that tolerate heat, ultraviolet irradiation ${ }^{113}$ and hydrogen-peroxide treatment. In addition, a self-cleaning capability could be implemented by changing the surface chemistry of the material or by coating materials with photothermal materials $^{32,109}$ or hydrophobic membranes ${ }^{31}$ to capture, kill and release the virus; for example, likewise, using filters composed of metal-organic frameworks with photodynamic ability ${ }^{109}$ or graphene with photothermal ability ${ }^{32}$. Functional nanofibres, for example, fibres modified with amino-functionalized silica particles ${ }^{114}$ 




Fig. 5 | Timeline of key contributions of materials science to virology.

FDA, US Food and Drug Administration; SHERLOCK, specific high-sensitivity enzymatic reporter unlocking. capture nucleic acids or proteins in a sample, which can then be separated by application of a magnetic field ${ }^{120}$. Integrating microfluidics with isolation techniques can reduce the amount of reagents, increase isolation speed and allow automation ${ }^{121}$. Moreover, microfluidics can be introduced to rapidly mass-synthesize uniform magnetic particles ${ }^{122}$ and to efficiently isolate viruses using magnetic-particle-based microfluidics ${ }^{123}$. In addition, DNA $^{124}$ or RNA nanostructures ${ }^{125}$ that contain complementary oligonucleotides to virus DNA or RNA could be combined with microfluidics for virus isolation. Although current nasal swabs are effective in collecting virus samples, nasal swabs with a controlled porous architecture could be functionalized with materials that can capture viruses through multivalent interactions ${ }^{119}$, which would enable the collection of viruses even at very low concentrations.

Similarly, aggregation-induced-emission (AIE) technology or quantum dots could be integrated with immune colloidal gold techniques to increase viral-detection sensitivity ${ }^{126}$. In addition, materials-science approaches, such as ionic-liquid-based materials ${ }^{127,128}$, could be applied to improve the stability of extracted nucleic acids or proteins for further investigation. Improving the sensitivity, specificity and simplicity of virus-detection methods is important to ensure accurate and rapid testing of samples. For example, an $\mathrm{Fe}-\mathrm{Au}$ nanoparticle-coupling approach, combining PCR amplification with the quantitative measurement of Au nanoparticles using inductively coupled plasma mass spectrometry ${ }^{129}$, which was originally developed for the detection of circulating tumour DNA, could also be applied for the sensitive and specific detection of SARS-CoV-2 RNA. Microarrays, which have been developed for the precise control and manipulation of fluids, combine sample preparation, reaction, separation and detection on one chip of a few square centimetres ${ }^{130}$, and could be used in combination with surface-enhanced Raman spectroscopy for rapid virus detection using nanoparticle aggregates or arrays that can generate surface-enhanced Raman spectroscopy signals ${ }^{131,132}$. Low-cost point-of-care devices, designed with microfluidics, could also be adapted for viral detection, requiring only small sample volumes. Microfluidics can further be used for the production of designed nanoparticles ${ }^{133,134}$, which are used for viral detection, treatment and vaccination.

Plasma protein profiles have been investigated as disease-related biomarkers using mass-spectroscopybased proteomics; similarly, viral protein biomarkers could be detected in blood ${ }^{135}$. However, the accuracy of protein identification by mass-spectrometry-based proteomics is limited, owing to the concentration range (from $\mathrm{pg} \mathrm{ml}^{-1}$ to $\mathrm{mg} \mathrm{ml}^{-1}$ ) of the $>10,000$ proteins in plasma. The protein corona, that is, the shell of biomolecules formed on a nanoparticle upon contact with a biofluid ${ }^{136}$, can be exploited to increase the concentration of proteins for mass spectrometry to overcome the bottleneck of blood-sample-based proteomics and to enable the detection of low-abundance proteins ${ }^{137}$. Therefore, investigating the protein corona ${ }^{138,139}$ may also provide a strategy for virus detection and/or to study the host response. For example, nanoparticles could be designed 
to adsorb viral protein biomarkers or host response proteins (that is, cytokines) in plasma, serum or other biological samples. The nanoparticles could then be isolated for downstream mass-spectrometry-based proteomics analysis, enabling fully automated, high-throughput viral detection, including nanoparticle-plasma incubation, protein-corona purification using magnetism, protein digestion and liquid chromatography-tandem mass spectrometry ${ }^{139}$.

Bioelectronic systems ${ }^{140}$ enable the real-time and remotely controlled monitoring of biological information in the body; for example, wearable sensors for the wireless monitoring of uric acid and tyrosine in sweat $^{141}$, glucose levels during diabetes therapy ${ }^{142}$ and skin temperature ${ }^{143}$. Bioelectronic devices could also be applied for the long-term monitoring of symptoms of viral infections, such as an increase in temperature or respiratory syndrome. Protective equipment could also be equipped with a sensor that can sense the entry of viral particles and translate the signal to a smartphone. For example, cellular-binding-like events between the virus and host-cell-mimicking sensors (that is, sensors coated with host-cell membranes or multivalent binders) can be detected by thermal-shift measurements based on protein stabilization induced by a ligand ${ }^{144,145}$, which can be translated into a digital signal recorded by a smartphone. Similarly, interactions between viral particles and specific molecules could be detected in protective masks.

Mobile technologies offer the possibility of track-andtrace systems that provide information on COVID-19infected patients, including age, gender, symptoms and geographical areas. These systems can alert users when they are in high-risk areas via an app to slow down the spread of SARS-CoV-2. Mobile technology can also be used to collect data for analysis and to inform guidelines for government policies. For example, a COVID-19 symptoms tracker app has been developed. The app has already collected data from over 2.8 million participants, providing information about risk factors, symptoms, clinical results and geographic hotspots. The data are publicly available and important in the response to a pandemic; however, privacy concerns and ethical considerations should also be considered ${ }^{146}$. In addition, AI, such as machine learning, can be applied to large datasets of clinical results to help clinicians in their diagnosis. For example, an AI system has been developed based on the CT database of COVID-19 patients, enabling rapid diagnosis $^{147}$.

Finally, point-of-care devices are particularly important in areas with low resources; for example, low-cost and portable gene-sequencing devices that can be operated without the need for trained medical staff. Accurate, real-time monitoring of viral transmission can further help to understand the infection process; for example, bioluminescent microscopy can be used to image the transmission of a virus with an inserted luciferase gene in mice ${ }^{148,149}$ or ferrets ${ }^{150}$. Similarly, fluorescent protein genes such as green fluorescent proteins and red fluorescent proteins genes ${ }^{151-153}$ could be integrated into the SARS-CoV-2 genome for tracking transmission in vitro and in animal models.
Drug discovery and delivery. Materials science can also contribute to the development of new technologies, such as thermal proximity coaggregation ${ }^{144}$ and time-resolved wide-angle $\mathrm{X}$-ray scattering ${ }^{154}$, which allow the dynamic observation of structural protein changes in host cells in response to a virus, and virus-drug interactions, to accelerate and improve the process of drug discovery. In addition, drug development relies heavily on studies in animal models, which cannot entirely recapitulate the human response and often inaccurately predict the safety and efficacy in humans. Alternatively, microphysiological systems, such as organ-on-a-chip platforms, in which engineered human tissues are connected by physiologically relevant flows, can be applied for drug development, as well as for toxicity and efficacy evaluation. Similarly, organoids derived from stem cells can provide macroscale models of human tissues featuring the complexity and spatial heterogeneity of human organs. However, compared with animal models, organs-on-a-chip and organoids would fail in discovering systemic immunity-modulation drugs, because they cannot recapitulate the organ microenvironment ${ }^{155,156}$. Moreover, the lifespan of these platforms would need to be prolonged to allow the generation of mature tissues undergoing homeostasis ${ }^{156}$. Importantly, organoids can be used for disease modelling and for the evaluation of antiviral drug toxicity and treatment effects ${ }^{157-159}$. For example, bat and human intestinal organoids were used to confirm that bat intestinal cells can be infected by SARS-CoV-2 and that the human intestinal tract is a possible transmission route of SARS-CoV-2 (REF. ${ }^{160}$ ). Furthermore, it could be demonstrated in SARS-CoV-2infected kidney and human blood vessel organoids that soluble human ACE2 can inhibit SARS-CoV-2 infection ${ }^{161}$. Materials science can improve organoid cultures by developing chemically defined, tunable, synthetic scaffolds that guide the assembly of human cells into tissues and that support organoid maturation ${ }^{162,163}$.

Nanoparticles can enable the effective delivery of drugs, improving circulation time, enhancing targeting efficiency and reducing potential adverse side effects. Nanocarriers can also be used to deliver antiviral therapeutics into cells to intervene with the viral-replication cycle at the molecular level or to deliver plasmid DNA ${ }^{164}$ and RRNA $^{165}$ for vaccination. Vaccine efficiency and safety are of the utmost importance, and, therefore, US Food and Drug Administration (FDA)-approved materials should be used as delivery platforms; for example, a lipid-nanoparticle-encapsulated mRNA, encoding the receptor binding domain of SARS-CoV-2, has been reported as a vaccine candidate ${ }^{166}$. The FDA-approved lipid nanoparticle improves the stability of the mRNA and the transfection efficiency in vivo.

Lessons learned from cancer vaccines can benefit the development of antiviral vaccines. Biocompatible materials have been explored for drug delivery in cancer, to improve the stability and pharmacokinetics of payloads and to prevent premature release or degradation. In addition, nanomaterials can be modified to target the delivery to antigen-presenting cells and to enable co-delivery of viral antigens and adjuvants to the lymph nodes ${ }^{167}$, for example, by using an albumin-hitchhiking 
approach ${ }^{168}$ or by targeting phagocytic cells to promote lymph-node transportation ${ }^{169}$. Nanomaterials can also be designed to respond to a specific environment or to external stimuli, allowing the control of payload release and preventing undesired immune reactions, such as immune toxicities in normal tissues caused by payload leakage or an immune cytokine storm caused by rapid payload release ${ }^{87}$, for example, $\mathrm{pH}$-responsive nanoparticles ${ }^{170}$ or light-responsive nanoparticles ${ }^{171}$. Cancer vaccines can be designed to integrate diagnosis and treatment using nanomaterials with imaging abilities $^{172}$, for example, to monitor the delivery of antigens and adjuvants in vivo, which may also be relevant for antiviral vaccines. Real-time data on the location and treatment responses could be generated by introducing NIR-II bioluminescence ${ }^{173}$, NIR-II chemiluminescence ${ }^{174}$ or NIR-II fluorescence ${ }^{175}$ to enable the rational design of nanoscale materials and bench-to-bedside translation of vaccines. Materials science also enables the design of self-administration platforms; for example, vaccines formulated as microneedle patches ${ }^{176}$, which slowly release payloads upon self-administration. Reproducibility and scalability also play major roles for the translation of nanomaterials for drug delivery. Microfluidic systems can be used for the controlled, reproducible and high-throughput synthesis of libraries of nanoparticles. Nanomaterials also play a role in virus treatment, for example, to provide cofactors, such as $\mathrm{Zn}^{2+}$, which inhibits RNA polymerase and, thus, transcription ${ }^{177}$. Zn-based nanomaterials that bind RNA polymerase could also be designed and administered to inhibit virus replication. In contrast to dendrimers or polymers, the valency and spatial structures of DNA nanostructures can be easily designed. In addition, DNA nanomaterials are non-toxic and stable. Therefore, DNA nanostructures with particular shapes, modified with virus-targeting aptamers, can be applied for virus detection and inhibition ${ }^{124}$. Owing to their customized spatial structure, such DNA nanostructures precisely match the viral surface, enabling efficient virus capture.

Inhalation as a route of drug and vaccine administration is particularly relevant for viruses causing respiratory symptoms, such as SARS-CoV-2, because it allows small-molecule drugs ${ }^{178}$ and vaccines ${ }^{179}$ to directly target the lungs, in which the virus resides ${ }^{180}$. Inhalation is particularly advantageous in resource-poor regions, because no medical staff is needed and self-administration of drugs or vaccines improves patient compliance. Inspired by the approved intranasal spray vaccine FluMist ${ }^{105}$, similar inhaled drugs or vaccines could be encapsulated using smart nanoparticles to promote their stability and to realize long-term and controlled release. In addition, inhaled nanoparticles encapsulating drugs or vaccines can be modified with specific antibodies targeting the cells in which the viruses reside to kill the viruses, or immune cells to trigger an immune response. This strategy could also decrease potential side effects of drugs and vaccines by avoiding drug delivery to healthy tissues. For example, nanomaterials could be coated with a specific ligand that binds to receptors on host cells of the virus in the lungs. Therefore, following inhalation, the nanoparticles would inhibit virus-host cell interactions.
In the case of SARS-CoV-2, the ACE2 receptor or the serine protease TMPRSS2 could be targeted ${ }^{68,181}$. Nanoparticles can also be modified with elements of host-cell membranes to inhibit viral entry ${ }^{64,66}$; for example, nanomaterials can be decorated with host-cell membranes derived from human lung epithelial type II cells or human macrophages ${ }^{62}$ to capture SARS-CoV-2 and inhibit infection. Similarly, using nanomaterials that can generate free radicals ${ }^{56}$ or heat ${ }^{32}$ could improve the antiviral efficacy of such approaches.

Nanoscale materials have a similar size to viruses, which allows them to interact with the virus at the same size level. Cyclodextrins modified with mercaptoundecane sulfonic acids ${ }^{59}$, nanoparticles modified with long linkers that mimic heparan sulfate proteoglycans ${ }^{60}$, nanodecoys ${ }^{64}$, cellular nanosponges ${ }^{62}$ or DNA nanostructures ${ }^{124}$ could bind the virus to inhibit infection by inhibiting virus entry ${ }^{56}$.

Viral infections, such as SARS-CoV ${ }^{182}$ and dengue ${ }^{183}$ virus, can cause cytokine storms as a result of an excessive immune response ${ }^{184}$. SARS-CoV-2 infection can also induce a cytokine storm in some patients, which contributes to inflammation and pneumonia ${ }^{185}$. Lessons from nanotechnology-based cancer immunotherapy can inform treatment designs to modulate the cytokine storm by delivering anti-inflammatory drugs or inhibitors to inflammation sites. In particular, bioresponsive nanomaterials ${ }^{186-188}$ that target inflammation sites can deliver anti-inflammatory drugs and inhibitors. However, the targeting efficacy and payload leakage still need to be optimized to decrease side effects. In addition, the fabrication of clinical-grade materials with the ability of modulating cytokine storms remains a challenge.

Medical equipment and instruments. Materials science can be applied to optimize filters in ventilators with self-cleaning and virus-inhibition ability. 3D printing of ventilators could be introduced and optimized to fabricate ventilators with more functions. Similarly, ECMO machines, which are applied as life-saving treatment for severe respiratory diseases, such as COVID-19, suffer from high complexity, the need for highly trained staff, low portability and concerns about potential harm ${ }^{189}$, such as changes in thrombotic ${ }^{190}$ and immune-related factors $^{189}$. A small device could be developed that combines the blood pump and the oxygenator to improve portability and reduce thrombotic issues, by decreasing the time that blood spends outside the body. Indeed, portable ECMO devices, such as the Maquet Cardiohelp device, which combines the pump and oxygenator in one unit ${ }^{191}$, have been developed. To decrease the size of such devices, wireless technology and energy-converting materials could be applied. In addition, bioelectronic systems and wireless mobile technology could be integrated in ECMO devices to monitor blood-status changes in real time. The gas-exchange membranes, which are a key component of ECMO machines, are usually made of hollow fibres, such as silicone rubber fibres ${ }^{192}$ or polymethylpentene materials ${ }^{193}$. Their resistance to blood flow and their gas-exchange capacity could be further improved by using $3 \mathrm{D}$-printable biocompatible materials, such as respiratory-membrane-derived materials. 


\section{Outlook}

Viral-disease outbreaks require a rapid response to obtain basic knowledge, but also continuous antiviral research. Materials science plays an important role in many aspects of antiviral research, including the study of viral biology and transmission, protection, identification of infected patients, treatment and vaccination. The investigation of new viral strains requires virus isolation and purification, size, structure and component determination, and analysis of infection, replication and spread. To prepare for new viral outbreaks and to accelerate and coordinate our response, portable and inexpensive devices for viral isolation and purification from samples such as blood or saliva are needed; for example, a hand-powered ultra-low-cost centrifuge ${ }^{194,195}$. To investigate virus structure and composition, electron microscopy, cryo-electron microscopy, confocal microscopy and sequencing technologies need to be applied. Additionally, the real-time dynamics of the replication of a single virus in host cells and tissues, including RNA replication, protein formation and cell-to-cell transmission, need to be assessed, for example, using super-resolution imaging or time-resolved wide-angle $\mathrm{X}$-ray scattering. Advanced materials-enabled technologies, such as thermal-proximity coaggregation ${ }^{144}$ and time-resolved wide-angle X-ray scattering ${ }^{154}$, could further enable the observation of interactions between drugs and viruses. These technologies will require more powerful instruments with high temporal and spatial resolution, as well as high sensitivity. However, spatial resolution should not be achieved at the expense of time resolution, for example, in super-resolution microscopy. The development of portable and inexpensive imaging equipment, such as the Octopi ${ }^{196}$, BiteOscope ${ }^{197}$ and scale-free vertical tracking microscopy ${ }^{198}$, are particularly important in low-resource settings. However, the nanoscale settings in viral research provide a challenge for portable optical-imaging systems, owing to the diffraction limit. Thus, the time resolution might be more important than the spatial resolution in this case.

During viral outbreaks, large amounts of masks and personal protective equipment need to be rapidly produced, for example, using $3 \mathrm{D}$ printing ${ }^{117}$ and nano-electrospinning for the fabrication of nanofibres to compose filters. Virus detection further needs to be quick and done with high accuracy and at a low price, for example, by distributing self-testing kits, which have a sample-collection tool for viral capture (enabled by a specific surface chemistry, for example, a coating that can capture viruses by multivalent interactions ${ }^{119}$ at low concentrations), and the results are shown on a strip ${ }^{43,199}$. Sharing of results and data is also an important aspect in antiviral research, in particular, during viral-disease outbreaks.

The delivery of antiviral drugs and vaccines will greatly benefit from the use of vehicles based on biocompatible materials to reduce systemic toxicity, to improve circulation time, for the co-delivery of multiple components, to increase drug or vaccine stability and to target specific cells or tissues. Drugs and vaccines need to be preclinically tested in cells and virus-infected animal models. Alternatively, engineered ex vivo systems can be applied, for example, organoids and organs-on-a-chip, which allow the assessment of drug effects in real time. The production of drugs or vaccines may further require the use of robots and microfluidic technologies to enable scalability and to be able to respond to high demand. To tackle future challenges, the collaboration between different scientific fields, clinicians and industry will be required.

Published online 14 October 2020
1. Gates, B. Responding to Covid-19 - a once-ina-century pandemic? N. Engl. J. Med. 382 1677-1679 (2020)

2. World Health Organization. Weekly operational update on COVID-19. 9 September 2020 (WHO, 2020).

3. Johns Hopkins University. COVID-19 dashboard by the center for systems science and engineering (CSSE) at Johns Hopkins University (JHU). JHU https://coronavirus.jhu.edu/map.html (2020).

4. The Novel Coronavirus Pneumonia Emergency Response Epidemiology Team. Vital surveillances: the epidemiological characteristics of an outbreak of 2019 novel coronavirus diseases (COVID-19) - China 2020. China CDC Wkly 2, 113-122 (2020).

5. Thorp, H. H. Time to pull together. Science 367, 1282 (2020).

6. Lauring, A. S., Frydman, J. \& Andino, R. The role of mutational robustness in RNA virus evolution. Nat. Rev. Microbiol. 11, 327-336 (2013).

7. Zhang, B. et al. Diagnosis of Zika virus infection on a nanotechnology platform. Nat. Med. 23, 548-550 (2017).

In this paper, a multiplexed assay on nanostructured plasmonic gold is used to study Zika virus infection.

8. Ferguson, B. S. et al. Genetic analysis of $\mathrm{H} 1 \mathrm{~N} 1$ influenza virus from throat swab samples in a microfluidic system for point-of-care diagnostics. J. Am. Chem. Soc. 133, 9129-9135 (2011).

9. Nguyen, D. N., Green, J. J., Chan, J. M., Langer, R. \& Anderson, D. G. Polymeric materials for gene delivery and DNA vaccination. Adv. Mater. 21, 847-867 (2009).

10. Gates, B. The next epidemic - lessons from Ebola N. Engl. J. Med. 372, 1381-1384 (2015).
11. Brandenburg, B. \& Zhuang, X. Virus traffickinglearning from single-virus tracking. Nat. Rev. Microbiol. 5, 197-208 (2007). This review summarizes the technical developments that have enabled single-virus imaging in live cells and insights into viral infection.

12. Lakadamyali, M., Rust, M. J., Babcock, H. P. \& Zhuang, X. Visualizing infection of individual influenza viruses. Proc. Natl Acad. Sci. USA 100, 9280-9285 (2003).

13. Deamer, D., Akeson, M. \& Branton, D. Three decades of nanopore sequencing. Nat. Biotechnol. 34 518-524 (2016) This perspective discusses the history of nanopore sequencing.

14. Drenth, J. Principles of Protein X-ray Crystallography (Springer, 2007).

15. Wuthrich, K. Protein structure determination in solution by nuclear magnetic resonance spectroscopy. Science 243, 45-50 (1989).

16. Cheng, Y., Grigorieff, N., Penczek, P. A. \& Walz, T. A primer to single-particle cryo-electron microscopy. Cell 161, 438-449 (2015).

17. Sirohi, D. et al. The 3.8 A resolution cryo-EM structure of Zika virus. Science 352, 467-470 (2016).

18. Wrapp, D. et al. Cryo-EM structure of the 2019-nCoV spike in the prefusion conformation. Science 367 , 1260-1263 (2020). This paper reports the structure of the SARS-CoV-2 spike protein using cryo-electron microscopy.

19. Killingley, B. \& Nguyen-Van-Tam, J. Routes of influenza transmission. Influenza Other Respir. Viruses 7, 42-51 (2013).

20. Abe, K. \& Inchauspe, G. Transmission of hepatitis C by saliva. Lancet 337, 248 (1991).
21. Royce, R. A., Sena, A., Cates, W. Jr \& Cohen, M. S Sexual transmission of HIV. N. Engl. J. Med. 336 1072-1078 (1997).

22. Cowling, B. J. et al. Aerosol transmission is an important mode of influenza A virus spread. Nat. Commun. 4, 1935-1940 (2013).

23. Liu, Y. et al. Evolutionary enhancement of Zika virus infectivity in Aedes aegypti mosquitoes. Nature 545, 482-486 (2017)

24. Acheson, D. \& Fiore, A. E. Hepatitis A transmitted by food. Clin. Infect. Dis. 38, 705-715 (2004).

25. Ward, J. W. et al. Transmission of human immunodeficiency virus (HIV) by blood transfusions screened as negative for HIV antibody. N. Engl. J. Med. 318, 473-478 (1988).

26. Sanders, J. M., Monogue, M. L., Jodlowski, T. Z. $\&$ Cutrell, J. B. Pharmacologic treatments for coronavirus disease 2019 (COVID-19): a review. JAMA 323, 1824-1836 (2020).

27. Shang, J. et al. Cell entry mechanisms of SARS-CoV-2 Proc. Natl Acad. Sci. USA 117, 11727-11734 (2020).

28. Shi, J. et al. Susceptibility of ferrets, cats, dogs, and other domesticated animals to SARS-coronavirus 2 Science 368, 1016-1020 (2020).

29. Sia, S. F. et al. Pathogenesis and transmission of SARS-CoV-2 in golden hamsters. Nature $\mathbf{5 8 3}$ 834-838 (2020).

30. Chua, M. H. et al. Face masks in the new COVID-19 normal: materials, testing, and perspectives. Research 2020, 7286735 (2020).

31. El-Atab, N., Qaiser, N., Badghaish, H. S., Shaikh, S. F. $\&$ Hussain, M. M. A flexible nanoporous template for the design and development of reusable antiCOVID-19 hydrophobic face masks. ACS Nano 14 , 7659-7665 (2020). 
32. Zhong, $\mathrm{H}$. et al. Reusable and recyclable graphene masks with outstanding superhydrophobic and photothermal performances. ACS Nano 14 6213-6221 (2020)

33. Liu, C. et al. Transparent air filter for high-efficiency $\mathrm{PM}_{25}$ capture. Nat. Commun. 6, 6205 (2015).

34. Zhang, G.-H. et al. High-performance particulate matter including nanoscale particle removal by a self-powered air filter. Nat. Commun. 11, 1653 (2020).

35. Panet, A., Baltimore, D. \& Hanafusa, T. Quantitation of avian RNA tumor virus reverse transcriptase by radioimmunoassay. J. Virol. 16, 146-152 (1975).

36. Clark, M. F. \& Adams, A. Characteristics of the microplate method of enzyme-linked immunosorben assay for the detection of plant viruses. J. Gen. Virol. 34, 475-483 (1977)

37. Faulk, W. P. \& Taylor, G. M. Communication to the editors: an immunocolloid method for the electron microscope. Immunochemistry 8, 1081-1083 (1971).

38. Patolsky, F. et al. Electrical detection of single viruses. Proc. Natl Acad. Sci. USA 101, 14017-14022 (2004).

39. Chowdhury, A. D., Takemura, K., Li, T.-C., Suzuki, T. $\&$ Park, E. Y. Electrical pulse-induced electrochemical biosensor for hepatitis $\mathrm{E}$ virus detection. Nat. Commun 10, 3737 (2019)

40. Carr, M. J. et al. Development of a real-time RT-PCR for the detection of swine-lineage influenza $A(\mathrm{H} 1 \mathrm{~N} 1)$ virus infections. J. Med. Virol. 45, 196-199 (2009).

41. Centers for Disease Control and Prevention. Interim guidelines for collecting, handling, and testing clinical specimens from persons for coronavirus disease 2019 (COVID-19) (CDC, 2020).

42. East-Seletsky, A. et al. Two distinct RNase activities of CRISPR-C2C2 enable guide-RNA processing and RNA detection. Nature 538, 270-273 (2016).

43. Broughton, J. P. et al. CRISPR-Cas12-based detection of SARS-CoV-2. Nat. Biotechnol. 38, 870-874 (2020).

44. Gootenberg, J. S. et al. Nucleic acid detection with CRISPR-Cas 13a/C2c2. Science 356, 438-442 (2017).

45. Kellner, M. J., Koob, J. G., Gootenberg, J. S. Abudayyeh, O. O. \& Zhang, F. SHERLOCK: nucleic acid detection with CRISPR nucleases. Nat. Protoc. 14 2986-3012 (2019).

46. Abbott, T. R. et al. Development of CRISPR as an antiviral strategy to combat SARS-CoV-2 and influenza. Cell 181, 856-876 (2020).

47. Baaske, M. D., Foreman, M. R. \& Vollmer, F. Single-molecule nucleic acid interactions monitored on a label-free microcavity biosensor platform. Nat. Nanotechnol. 9, 933-939 (2014).

48. Wang, M. et al. Nanopore targeted sequencing for the accurate and comprehensive detection of SARS-CoV-2 and other respiratory viruses. Small 16, 2002169 (2020).

49. Van Herwaarden, A. \& Sarro, P. Thermal sensors based on the Seebeck effect. Sens. Actuators 10, 321-346 (1986)

50. Chung, M. et al. CT imaging features of 2019 novel coronavirus (2019-nCoV). Radiology 295, 202-207 (2020).

51. Chen, S.-C., Tsai, T.-T. \& Luo, C.-H. Portable and programmable clinical EOG diagnostic system. J. Med. Eng. Technol. 24, 154-162 (2000).

52. Sands, V. M., Auerbach, P. S., Birnbaum, J. \& Green, M Evaluation of a portable clinical blood analyzer in the emergency department. Acad. Emerg. Med. 2 172-178 (1995)

53. Li, L. et al. Artificial intelligence distinguishes COVID-19 from community acquired pneumonia on chest CT. Radiology 296, E65-E71 (2020).

54. De Clercq, E. \& Herdewijn, P. in Pharmaceutical Sciences Encyclopedia: Drug Discovery, Development and Manufacturing (Wiley, 2010).

55. De Clercq, E. Antivirals and antiviral strategies. Nat. Rev. Microbiol. 2, 704-720 (2004).

56. Vigant, F., Santos, N. C. \& Lee, B. Broad-spectrum antivirals against viral fusion. Nat. Rev. Microbiol. 13 426-437 (2015)

57. Wang, M. et al. Remdesivir and chloroquine effectively inhibit the recently emerged novel coronavirus (2019-nCoV) in vitro. Cell Res. 30, 269-271 (2020)

58. De Clercq, E. \& Li, G. Approved antiviral drugs over the past 50 years. Clin. Microbiol. Rev. 29, 695-747 (2016)

59. Jones, S. T. et al. Modified cyclodextrins as broadspectrum antivirals. Sci. Adv. 6, eaax9318 (2020)

60. Cagno, V. et al. Broad-spectrum non-toxic antiviral nanoparticles with a virucidal inhibition mechanism Nat. Mater. 17, 195-203 (2018).
61. Lauster, D. et al. Phage capsid nanoparticles with defined ligand arrangement block influenza virus entry. Nat. Nanotechnol. 15, 373-379 (2020).

62. Zhang, Q. et al. Cellular nanosponges inhibit SARS-CoV-2 infectivity. Nano Lett. 20, 5570-5574 (2020).

63. Fang, R. H., Kroll, A. V., Gao, W. \& Zhang, L. Cell membrane coating nanotechnology. Adv. Mater. 30 1706759 (2018)

64. Rao, L., Tian, R. \& Chen, X. Cell-membrane-mimicking nanodecoys against infectious diseases. ACS Nano 14 2569-2574 (2020)

65. Zhang, P. et al. Virus-mimetic nanovesicles as a versatile antigen-delivery system. Proc. Natl Acad. Sci. USA 112, E6129-E6138 (2015)

66. Rao, L. et al. A biomimetic nanodecoy traps zika virus to prevent viral infection and fetal microcephaly development. Nano Lett. 19, 2215-2222 (2018).

67. Zhang, Q. et al. Neutrophil membrane-coated nanoparticles inhibit synovial inflammation and alleviate joint damage in inflammatory arthritis. Nat. Nanotechnol. 13, 1182-1190 (2018).

68. Hoffmann, M. et al. SARS-CoV-2 cell entry depends on ACE2 and TMPRSS2 and is blocked by a clinically proven protease inhibitor. Cell 181, 271-280 (2020).

69. Grein, J. et al. Compassionate use of remdesivir for patients with severe Covid-19. N. Engl. J. Med. 384 2327-2336 (2020).

70. RECOVERY Collaborative Group et al. Dexamethasone in hospitalized patients with Covid-19 - preliminary report. N. Engl. J. Med. https://doi.org/10.1056/ NEJMoa2021436 (2020)

71. Lammers, T. et al. Dexamethasone nanomedicines for COVID-19. Nat. Nanotechnol. 15, 622-624 (2020).

72. Wu, Y. et al. A noncompeting pair of human neutralizing antibodies block COVID-19 virus binding to its receptor ACE2. Science 368, 1274-1278 (2020).

73. Cao, Y. et al. Potent neutralizing antibodies against SARS-CoV-2 identified by high-throughput single-cel sequencing of convalescent patients' B cells. Cell 181, 73-84 (2020).

74. Shi, R. et al. A human neutralizing antibody targets the receptor binding site of SARS-CoV-2. Nature 584 120-124 (2020)

75. Walter, J. D. et al. Synthetic nanobodies targeting the SARS-CoV-2 receptor-binding domain. Preprint at BioRxiv https://doi.org/10.1101/2020.04.16.045419 (2020).

76. Wrapp, D. et al. Structural basis for potent neutralization of betacoronaviruses by single-domain camelid antibodies. Cell 181, 1004-1015 (2020).

77. Zhu, F.-C. et al. Safety, tolerability, and immunogenicity of a recombinant adenovirus type- 5 vectored COVID-19 vaccine: a dose-escalation, openlabel, non-randomised, first-in-human trial. Lancet 395, 1845-1854 (2020)

78. US National Library of Medicine. ClinicalTrials.gov https://clinicaltrials.gov/ct2/show/NCT04313127 (2020).

79. Gao, Q. et al. Development of an inactivated vaccine candidate for SARS-CoV-2. Science 369, 77-81 (2020).

80. Hotez, P. J., Corry, D. B., Strych, U. \& Bottazzi, M. E. COVID-19 vaccines: neutralizing antibodies and the alum advantage. Nat. Rev. Immunol. 20, 399-400 (2020).

81. Burton, D. R. Antibodies, viruses and vaccines. Nat. Rev. Immunol. 2, 706-713 (2002)

82. Ryan, K. J. \& Ray, C. G. Medical microbiology. McGraw Hill 4, 370 (2004).

83. Wang, J. et al. Pulmonary surfactant-biomimetic nanoparticles potentiate heterosubtypic influenza immunity. Science 367, eaau0810 (2020). Pulmonary surfactant-biomimetic nanoparticles are used to initiate broad-spectrum antiviral immunity

84. Herold S, \& Sander, L.-E. Toward a universal flu vaccine. Science 367, 852-853 (2020).

85. Coffman, R. L., Sher, A. \& Seder, R. A. Vaccine adjuvants: putting innate immunity to work. Immunity 33, 492-503 (2010)

86. Pardi, N., Hogan, M. J., Porter, F. W. \& Weissman, D. mRNA vaccines - a new era in vaccinology. Nat. Rev. Drug Discov. 17, 261-279 (2018).

87. Riley, R. S., June, C. H., Langer, R. \& Mitchell, M. J. Delivery technologies for cancer immunotherapy. Nat. Rev. Drug Discov. 18, 175-196 (2019).

88. Kong, N. et al. Synthetic mRNA nanoparticlemediated restoration of p53 tumor suppressor sensitizes p53-deficient cancers to mTOR inhibition Sci. Transl. Med 11, eaaw1565 (2019).
89. Richner, J. M. et al. Modified mRNA vaccines protect against Zika virus infection. Cell 168, 1114-1125. e1110 (2017)

In this paper, the authors used a lipid nanoparticle to deliver an mRNA vaccine against Zika virus infection.

90. US National Library of Medicine. ClinicalTrials.gov https://clinicaltrials.gov/ct2/show/NCT04368728 (2020)

91. Vogel, A. et al. A prefusion SARS-CoV-2 spike RNA vaccine is highly immunogenic and prevents lung infection in non-human primates. Preprint at bioRxiv https://doi.org/10.1101/2020.09.08.280818 (2020)

92. US National Library of Medicine. Clinicaltrials.gov https://clinicaltrials.gov/ct2/show/NCT04232280 (2020)

93. US National Library of Medicine. ClinicalTrials.gov https://clinicaltrials.gov/ct2/show/NCT04470427 (2020)

94. Mustafa, A. K. et al. Extracorporeal membrane oxygenation for patients with COVID-19 in severe respiratory failure. JAMA Surg. https://doi.org/ 10.1001/jamasurg.2020.3950 (2020).

95. Brodie, D. \& Bacchetta, M. Extracorporeal membrane oxygenation for ARDS in adults. N. Engl. J. Med. 365 1905-1914 (2011).

96. Ivanovsky, D. Über die Mosaikkrankheit der Tabakspflanze. St. Petersb. Acad. Imp. Sci. Bull. 35 67-70 (1892)

97. Drinker, P. \& Shaw, L. A. The prolonged administration of artificial respiration. J. Franklin Inst. 213, 355-372 (1932).

98. Knoll, M. \& Ruska, E. Das elektronenmikroskop. Z. Phys. 78, 318-339 (1932).

99. Ruska, H. Die Sichtbarmachung der bakteriophagen lyse im übermikroskop. Naturwissenschaften $\mathbf{2 8}$ 45-46 (1940).

100. Franks, A. An optically focusing X-ray diffraction camera. Proc. Phys. Soc. B 68, 1054 (1955).

101. US Food and Drug Administration. Drugs@FDA: FDA approved drugs. FDA https://www.accessdata.fda.gov/ scripts/cder/daf/index.cfm? event=reportsSearch. process (1963).

102. Rashkind, W., Freeman, A., Klein, D. \& Toft, R. Evaluation of a disposable plastic, low volume, pumpless oxygenator as a lung substitute. J. Pediatr. 66, 94-102 (1965).

103. Schena, M., Shalon, D., Davis, R. W. \& Brown, P. O Quantitative monitoring of gene expression patterns with a complementary DNA microarray. Science $\mathbf{2 7 0}$ 467-470 (1995).

104. US Food and Drug Administration. Highlights of prescribing information Doxil (FDA, 1995).

105. US Food and Drug Administration. FDA information regarding FluMist quadrivalent vaccine. FDA https:/ www.fda.gov/vaccines-blood-biologics/vaccines/ fda-information-regarding-flumist-quadrivalent-vaccine (2018).

106. Eisenstein, M. Oxford Nanopore announcement sets sequencing sector abuzz. Nat. Biotechnol. 30 , 295-296 (2012).

107. Murray, B. K. et al. Virion disruption by ozonemediated reactive oxygen species. J. Virol. Methods 153, 74-77 (2008).

108. Almeida, A., Faustino, M. A. F. \& Neves, M. G. Antimicrobial photodynamic therapy in the control of COVID-19. Antibiotics 9, 320 (2020).

109. Li, P. et al. Metal-organic frameworks with photocatalytic bactericidal activity for integrated air cleaning. Nat. Commun. 10, 2177 (2019).

110. Centers for Disease Control and Prevention. Implementing filtering facepiece respirator (FFR) reuse, including reuse after decontamination, when there are known shortages of N95 respirators (CDC, 2020).

111. Koo, W-T. et al. Hierarchical metal-organic framework-assembled membrane filter for efficient removal of particulate matter. ACS Appl. Mater. Interfaces 10, 19957-19963 (2018)

112. Hao, Z., Wu, J., Wang, C. \& Liu, J. Electrospun polyimide/metal-organic framework nanofibrous membrane with superior thermal stability for efficient $\mathrm{PM}_{25}$ capture. ACS Appl. Mater. Interfaces 11, 11904-11909 (2019).

113. Liao, L. et al. Can N95 respirators be reused after disinfection? How many times? ACS Nano 14 6348-6356 (2020).

114. Chen, Z., Hsu, F.-C., Battigelli, D. \& Chang, H.-C. Capture and release of viruses using aminofunctionalized silica particles. Anal. Chim. Acta 569 76-82 (2006) 
115. Badrossamay, M. R., Mcllwee, H. A., Goss, J. A \& Parker, K. K. Nanofiber assembly by rotary jet-spinning. Nano Lett. 10, 2257-2261 (2010).

116. Murphy, S. V. \& Atala, A. 3D bioprinting of tissues and organs. Nat. Biotechnol. 32, 773-785 (2014).

117. Choong, Y. Y. C. et al. The global rise of 3D printing during the COVID-19 pandemic. Nat. Rev. Mater. $\mathbf{5}$ 637-639 (2020)

118. Ong, S. W. X. et al. Air, surface environmental, and personal protective equipment contamination by severe acute respiratory syndrome coronavirus 2 (SARS-CoV-2) from a symptomatic patient. JAMA 323 1610-1612 (2020)

119. Dey, P. et al. Multivalent flexible nanogels exhibit broad-spectrum antiviral activity by blocking virus entry. ACS Nano 12, 6429-6442 (2018).

120. Berensmeier, S. Magnetic particles for the separation and purification of nucleic acids. Appl. Microbiol. Biotechnol. 73, 495-504 (2006).

121. Reinholt, S. J. \& Baeumner, A. J. Microfluidic isolation of nucleic acids. Angew. Chem. Int. Ed. 53, 13988-14001 (2014).

122. Taddei, C. et al. Fabrication of polystyreneencapsulated magnetic iron oxide nanoparticles via batch and microfluidic-assisted production. Colloid Polym. Sci. 297, 861-870 (2019).

123. Unni, M. et al. Engineering magnetic nanoparticles and their integration with microfluidics for cell isolation. J. Colloid Interface Sci. 564, 204-215 (2020).

124. Kwon, P. S. et al. Designer DNA architecture offers precise and multivalent spatial pattern-recognition for viral sensing and inhibition. Nat. Chem. 12, 26-35 (2020).

125. Geary, C., Rothemund, P. W. \& Andersen, E. S A single-stranded architecture for cotranscriptional folding of RNA nanostructures. Science 345, 799-804 (2014).

126. Nankai University. Fast testing only Takes 15 minutes: Nankai University's team developed antibody test kit for novel coronavirus. Nankai Univ. http://en. nankai. edu.cn/2020/0219/c14577a265299/page.htm (2020)

127. Singh, N., Sharma, M., Mondal, D., Pereira, M. M. $\&$ Prasad, K. Very high concentration solubility and long-term stability of DNA in an ammonium-based ionic liquid: A suitable medium for nucleic acid packaging and preservation. ACS Sustain. Chem. Eng. 5, 1998-2005 (2017).

128. Zhu, C., Varona, M. \& Anderson, J. L. Magnetic ionic liquids as solvents for RNA extraction and preservation ACS Omega 5, 11151-11159 (2020).

129. Hu, P. et al. Fe-Au nanoparticle-coupling for ultrasensitive detections of circulating tumor DNA. Adv. Mater. 30, 1801690 (2018)

130. Wang, D. et al. Microarray-based detection and genotyping of viral pathogens. Proc. Natl Acad. Sci. USA 99, 15687-15692 (2002).

131. Lim, D.-K. et al. Highly uniform and reproducible surface-enhanced Raman scattering from DNAtailorable nanoparticles with 1-nm interior gap. Nat. Nanotechnol. 6, 452-460 (2011).

132. Di Zhang, L. H., Liu, B., Ge, Q., Dong, J. \& Zhao, X Rapid and ultrasensitive quantification of multiplex respiratory tract infection pathogen via lateral flow microarray based on SERS nanotags. Theranostics 9 . 4849-4859 (2019).

133. Rao, L. et al. A microfluidic electrostatic separator based on pre-charged droplets. Sens. Actuators $B$ Chem. 210, 328-335 (2015).

134. Rao, L. et al. Microfluidic electroporation-facilitated synthesis of erythrocyte membrane-coated magnetic nanoparticles for enhanced imagingguided cancer therapy. ACS Nano 11, 3496-3505 (2017).

135. Sánchez-Purrà, M. et al. Surface-enhanced Raman spectroscopy-based sandwich immunoassays for multiplexed detection of Zika and Dengue viral biomarkers. ACS Infect. Dis. 3, 767-776 (2017).

136. Bertrand, N. et al. Mechanistic understanding of in vivo protein corona formation on polymeric nanoparticles and impact on pharmacokinetics. Nat. Commun. 8, 777 (2017).

137. Hadjidemetriou, M., Al-Ahmady, Z., Buggio, M. Swift, J. \& Kostarelos, K. A novel scavenging tool for cancer biomarker discovery based on the bloodcirculating nanoparticle protein corona. Biomaterials 188, 118-129 (2019).

138. Pattipeiluhu, R. et al. Unbiased identification of the liposome protein corona using photoaffinity-based chemoproteomics. ACS Cent. Sci. 6, 535-545 (2020).
139. Blume, J. E. et al. Rapid, deep and precise profiling of the plasma proteome with multi-nanoparticle protein corona. Nat. Commun. 11, 3662 (2020).

140. Schmidt, C. Bioelectronics: The bionic material. Nature 483, S37 (2012).

141. Yang, Y. et al. A laser-engraved wearable sensor for sensitive detection of uric acid and tyrosine in sweat. Nat. Biotechnol. 38, 217-224 (2020).

142. Lee, H. et al. A graphene-based electrochemical device with thermoresponsive microneedles for diabetes monitoring and therapy. Nat. Nanotechnol. 11 566-572 (2016)

143. Nakata, S., Arie, T., Akita, S. \& Takei, K. Wearable, flexible, and multifunctional healthcare device with an ISFET chemical sensor for simultaneous sweat $\mathrm{pH}$ and skin temperature monitoring. ACS Sens. 2, 443-448 (2017).

144. Tan, C. S. H. et al. Thermal proximity coaggregation for system-wide profiling of protein complex dynamics in cells. Science 359, 1170-1177 (2018).

145. Molina, D. M. et al. Monitoring drug target engagement in cells and tissues using the cellular thermal shift assay. Science 341, 84-87 (2013).

146. Drew, D. A. et al. Rapid implementation of mobile technology for real-time epidemiology of COVID-19. Science 368, 1362-1367 (2020).

147. Zhang, K. et al. Clinically applicable Al system for accurate diagnosis, quantitative measurements and prognosis of COVID-19 pneumonia using computed tomography. Cell 181, 1423-1433 (2020).

148. Pan, W. et al. Visualizing influenza virus infection in living mice. Nat. Commun. 4, 2369-2377 (2013).

149. Rameix-Welti, M.-A. et al. Visualizing the replication of respiratory syncytial virus in cells and in living mice. Nat. Commun. 5, 5104 (2014).

150. Karlsson, E. A. et al. Visualizing real-time influenza virus infection, transmission and protection in ferrets. Nat. Commun. 6, 6378 (2015).

151. Finke, S., Brzózka, K. \& Conzelmann, K.-K. Tracking fluorescence-labeled rabies virus: enhanced green fluorescent protein-tagged phosphoprotein P supports virus gene expression and formation of infectious particles. J. Virol. 78, 12333-12343 (2004).

152. Lux, K. et al. Green fluorescent protein-tagged adenoassociated virus particles allow the study of cytosolic and nuclear trafficking. J. Virol. 79, 11776-11787 (2005).

153. Klingen, Y., Conzelmann, K.-K. \& Finke, S. Double-labeled rabies virus: live tracking of enveloped virus transport. J. Virol. 82, 237-245 (2008).

154. Cammarata, M. et al. Tracking the structural dynamics of proteins in solution using time-resolved wide-angle X-ray scattering. Nat. Methods 5, 881-886 (2008).

155. Junttila, M. R. \& de Sauvage, F. J. Influence of tumour micro-environment heterogeneity on therapeutic response. Nature 501, 346-354 (2013).

156. Rossi, G., Manfrin, A. \& Lutolf, M. P. Progress and potential in organoid research. Nat. Rev. Genet. 19 671-687 (2018)

157. Zhou, T. et al. High-content screening in hPSC-neural progenitors identifies drug candidates that inhibit Zika virus infection in fetal-like organoids and adult brain. Cell Stem Cell 21, 274-283.e5 (2017).

158. Watanabe, M. et al. Self-organized cerebral organoids with human-specific features predict effective drugs to combat Zika virus infection. Cell Rep. 21, 517-532 (2017).

159. Duan, $X$. et al. Identification of drugs blocking SARS-CoV-2 infection using human pluripotent stem cell-derived colonic organoids. Preprint at bioRxiv https://doi.org/10.1101/2020.05.02.073320 (2020).

160. Zhou, J. et al. Infection of bat and human intestinal organoids by SARS-CoV-2. Nat. Med. 26, 1077-1083 (2020).

161. Monteil, V. et al. Inhibition of SARS-CoV-2 infections in engineered human tissues using clinical-grade soluble human ACE2. Cell 181, 905-913 (2020).

162. Aisenbrey, E. A. \& Murphy, W. L. Synthetic alternatives to Matrigel. Nat. Rev. Mater. 5, 539-551 (2020).

163. Kratochvil, M. J. et al. Engineered materials for organoid systems. Nat. Rev. Mater. 4, 606-622 (2019).

164. Wang, C. et al. Molecularly engineered poly(ortho ester) microspheres for enhanced delivery of DNA vaccines. Nat. Mater. 3, 190-196 (2004).

165. Miao, L. et al. Delivery of mRNA vaccines with heterocyclic lipids increases anti-tumor efficacy by STING-mediated immune cell activation. Nat. Biotechnol. 37, 1174-1185 (2019).

166. Zhang, N.-N. et al. A thermostable mRNA vaccine against COVID-19. Cell 182, 1271-1283 (2020).
167. John, A. L. S., Chan, C. Y., Staats, H. F., Leong, K. W. \& Abraham, S. N. Synthetic mast-cell granules as adjuvants to promote and polarize immunity in lymph nodes. Nat. Mater. 11, 250-257 (2012).

168. Liu, H. et al. Structure-based programming of lymphnode targeting in molecular vaccines. Nature 507 , 519-522 (2014).

169. Schudel, A., Francis, D. M. \& Thomas, S. N. Material design for lymph node drug delivery. Nat. Rev. Mater. 4, 415-428 (2019).

170. Wilson, J. T. et al. pH-Responsive nanoparticle vaccines for dual-delivery of antigens and immunostimulatory oligonucleotides. ACS Nano 7, 3912-3925 (2013).

171. Zhang, C. et al. A light responsive nanoparticle-based delivery system using pheophorbide A graft polyethylenimine for dendritic cell-based cancer immunotherapy. Mol. Pharm. 14, 1760-1770 (2017).

172. Zhong, Y. et al. In vivo molecular imaging for immunotherapy using ultra-bright near-infrared-Ilb rare-earth nanoparticles. Nat. Biotechnol. 37 1322-1331 (2019).

173. Lu, L. et al. NIR-II bioluminescence for in vivo high contrast imaging and in situ ATP-mediated metastases tracing. Nat. Commun. 11, 4192 (2020).

174. Yang, Y. et al. NIR-II chemiluminescence molecular sensor for in vivo high-contrast inflammation imaging Angew. Chem. Int. Ed. 59, 18380 (2020).

175. Li, B. et al. Organic NIR-II molecule with long blood half-life for in vivo dynamic vascular imaging. Nat. Commun. 11, 3102 (2020).

176. McHugh, K. J. et al. Biocompatible near-infrared quantum dots delivered to the skin by microneedle patches record vaccination. Sci. Transl. Med. 11, eaay7162 (2019).

177. Kaushik, N. et al. Zinc salts block hepatitis E virus replication by inhibiting the activity of viral RNA-dependent RNA polymerase. J. Virol. 91 , e00754-00717 (2017).

178. Boivin, G. et al. Rapid antiviral effect of inhaled zanamivir in the treatment of naturally occurring influenza in otherwise healthy adults. J. Infect. Dis. 181, 1471-1474 (2000)

179. Garcia-Contreras, L., Awashthi, S., Hanif, S. \& Hickey, A. Inhaled vaccines for the prevention of tuberculosis. J. Mycobac Dis. S1, 002 (2012).

180. Willis, L., Hayes, D. \& Mansour, H. M. Therapeutic liposomal dry powder inhalation aerosols for targeted lung delivery. Lung 190, 251-262 (2012).

181. Elmezayen, A. D., Al-Obaidi, A., Sahin, A. T. $\&$ Yelekçi, K. Drug repurposing for coronavirus (COVID-19): in silico screening of known drugs against coronavirus $3 \mathrm{CL}$ hydrolase and protease enzymes. J. Biomol. Struct. Dyn. https://doi.org/10.1080/ 07391102.2020 .1758791 (2020)

182. Huang, K. J. et al. An interferon- $\gamma$-related cytokine storm in SARS patients. J. Med. Virol. 75, 185-194 (2005).

183. Rothman, A. L. Immunity to dengue virus: a tale of original antigenic sin and tropical cytokine storms. Nat. Rev. Immunol. 11, 532-543 (2011).

184. Tisoncik, J. R. et al. Into the eye of the cytokine storm Microbiol. Mol. Biol. Rev. 76, 16-32 (2012)

185. Pedersen, S. F. \& Ho, Y.-C. SARS-CoV-2: a storm is raging. J. Clin. Invest. 130, 2202-2205 (2020).

186. Dormont, F. et al. Squalene-based multidrug nanoparticles for improved mitigation of uncontrolled inflammation in rodents. Sci. Adv. 6, eaaz5466 (2020).

187. Zhang, C. Y., Gao, J. \& Wang, Z. Bioresponsive nanoparticles targeted to infectious microenvironments for sepsis management. Adv. Mater. 30, e1803618 (2018).

188. Ma, Q. et al. Calming cytokine storm in pneumonia by targeted delivery of TPCA-1 using platelet-derived extracellular vesicles. Matter 3, 287-301 (2020).

189. Henry, B. M. COVID-19, ECMO, and lymphopenia: a word of caution. Lancet Respir. Med. 8, e24 (2020).

190. Chlebowski, M. M., Baltagi, S., Carlson, M., Levy, J. H. \& Spinella, P. C. Clinical controversies in anticoagulation monitoring and antithrombin supplementation for ECMO Crit Care 24, 19-30 (2020).

191. Tulman, D. B. et al. Veno-venous ECMO: a synopsis of nine key potential challenges, considerations, and controversies. BMC Anesthesiol. 14, 65 (2014).

192. Motomura, T. et al. Development of silicone rubber hollow fiber membrane oxygenator for ECMO. Artif. Organs 27, 1050-1053 (2003).

193. Agati, S. et al. DIDECMO: A new polymethylpentene oxygenator for pediatric extracorporeal membrane oxygenation. ASAIO J. 52, 509-512 (2006). 
194. Bhamla, M. S. et al. Hand-powered ultralow-cost paper centrifuge. Nat. Biomed. Eng. 1, 0009 (2017).

195. Li, E., Larson, A., Kothari, A. \& Prakash, M. Handyfuge-LAMP: low-cost and electricity-free centrifugation for isothermal SARS-CoV-2 detection in saliva. Preprint at medRxiv https://doi.org/ 10.1101/2020.06.30.20143255 (2020).

196. Li, H., Soto-Montoya, H., Voisin, M., Valenzuela, L. F \& Prakash, M. Octopi: open configurable highthroughput imaging platform for infectious disease diagnosis in the field. Preprint at bioRxiv https:// doi.org/10.1101/684423 (2019).

197. Hol, F. J., Lambrechts, L. \& Prakash, M. BiteOscope an open platform to study mosquito biting behavior eLife 9, e56829 (2020).

198. Krishnamurthy, D. et al. Scale-free vertical tracking microscopy. Nat. Methods 17, 1040-1051 (2020).

199. Zhang, F., Abudayyeh, O. O. \& Gootenberg, J. S. A protocol for detection of COVID-19 using CRISPR diagnostics (Broad Institute, 2020).

Acknowledgements

This study is supported by the US METAvivor Early Career Investigator Award (No. 2018A020560, W.Tao), Harvard Medical School/Brigham and Women's Hospital Department of Anaesthesiology Basic Scientist Grant (No. 2420 BPA075, W.Tao) and Center for Nanomedicine Research Fund (No.2019A014810, W.Tao). W.Tao is a recipient of the Khoury Innovation Award (No. 2020A003219) and American Heart Association (AHA) Collaborative Science Award (No. 2018A004190). W.Tao also received a start-up package (for 3 years) from the Department of Anesthesiology, Perioperative and Pain Medicine to establish his independent research laboratory at Harvard Medical School and Brigham and Women's Hospital. We thank our department for this generous support. We also thank J. Chen from University of California, Los Angeles, for his proofreading and correction before the online publication of this Review.

\section{Author contributions}

All authors contributed to the discussion and writing of this manuscript.

\section{Competing interests}

O.C.F. has financial interests in Selecta Biosciences, Tarveda Therapeutics and Seer. R.L. receives licensing fees (to patents on which he was an inventor) from, invested in, consults (or was on scientific advisory boards or boards of directors) for lectured (and received a fee) or conducts sponsored research at MIT for which he was not paid for the following entities: 7th Sense, Abpro Labs, Abpro Korea, Acorda (formerly Civita Therapeutics), Aleph Farms, Alivio Therapeutics, Alkermes, Allevi, Alnylam Pharmaceuticals, Apotex Arcadia Biosciences, Arsenal Medical, Artificial Cells, Avalon GloboCare BASF, Biolnnovation Institute, Blackstone, Boston Children's Hospital, Celero, Cellomics, Cellular Biomedical, Charles River, Clontech, Combined Therapeutics, Conference Forum, Cornell University, CRISPR Therapeutics AG, Crown Bioscience, Cygnal Therapeutics, Daros, Daré Bioscience, DeepBiome, Dewpoint Therapeutics, Dispendix/Cellink, Domain, Eagle, EdiGene Biotechnology, Editas Medicine, El Lily, Eisai, Entrega, Everlywell, Evox Therapeutics, Flagship Pioneering, Frequency Therapeutics, GeneMedicine,
GenScript USA, GENUV, GlaxoSmithKline, Glycobia, Glympse Bio, GreenLight Biosciences, HCR, Hopewell Therapeutics, Horizon Discovery, Humacyte, IBEX Pharmaceuticals, ImmuneXcite, Indivior, Inovio, Institute of Immunology, Integrated DNA Technologies, InVivo Therapeutics, IxBio, J.R. Simplot, Jnana Therapeutics, Kala Pharmaceuticals, Kallyope, Kensa, Kodikaz Therapeutics, KSO Therapeutics, Landsdowne Labs, LikeMinds, Luminopia, Luye, Lyndra Therapeutics, Lyra Therapeutics, McGovern Institute, Medikinetics, Merck, MGH Ragon Institute, Micelle, Moderna Therapeutics, Momenta, Monsanto, Muse Biotechnologies, Mylan, Nanobiosym, Nanobiotix, NewBridge Ventures, Noveome Biotherapeutics, Novo Nordisk, Particles for Humanity, Pfizer, Pioneer Hi-Bred International, Polaris Partners, Portal Instruments, Preceres, Pulmatrix, PureTech, ReLive, ReproCELL USA, Rubius Therapeutics, Secant Medical, Seer, Selecta Biosciences, Senses Biosciences, Senses, Setsuro Tech, Seventh Sense Biosystems, Shenzhen Rice Life Technology, Shire AG, Shiseido, Siglion, Sigma-Aldrich, SiO2, SKE S.R.L., Soil Culture Solutions, SQZ Biotechnologies, StemBioSys, Suono Bio, T2 Biosystems, Taconic Biosciences, TARA, Tarveda Therapeutics, Tesio Pharmaceuticals, Third Rock, Tiba Biotech, TISSIUM, TransGen, Translate Bio, TriLink BioTechnologies, Unilever, VasoRx, Verseau Therapeutics, Vivtex, Whitehead Institute, Wiki Foods, YZ Biosciences and Zenomics.

Publisher's note

Springer Nature remains neutral with regard to jurisdictional claims in published maps and institutional affiliations.

(C) Springer Nature Limited 2020 